\title{
Les explications métagraphiques appliquées aux premières écritures enfantines
}

\section{Jacques David}

\section{(2) OpenEdition \\ 1 Journals}

\section{Édition électronique}

URL : http://journals.openedition.org/pratiques/1230

DOI : $10.4000 /$ pratiques. 1230

ISSN : 2425-2042

\section{Éditeur}

Centre de recherche sur les médiations (CREM)

\section{Édition imprimée}

Date de publication : 15 décembre 2008

Pagination : 163-187

\section{Référence électronique}

Jacques David, «Les explications métagraphiques appliquées aux premières écritures enfantines », Pratiques [En ligne], 139-140 | 2008, mis en ligne le 15 décembre 2008, consulté le 08 mars 2021.

URL : http://journals.openedition.org/pratiques/1230; DOI : https://doi.org/10.4000/pratiques.1230

(C) Tous droits réservés 


\title{
Les explications métagraphiques appliquées aux premières écritures enfantines
}

\author{
Jacques David \\ Université de Cergy-Pontoise \\ IUFM département de français \& UFR de Lettres Sciences humaines \\ EA 1392 - Centre de recherche Textes et Francophonies (LaSCoD)
}

\section{Introduction}

La présente étude ${ }^{(1)} \mathrm{s}$ 'appuie sur un large corpus de textes écrits et surtout de commentaires ou d'explications métagraphiques produits par de jeunes apprentisscripteurs $(5-7 \text { ans })^{(2)}$. Elle entend montrer comment ils parviennent à verbaliser des savoirs émergents sur le fonctionnement de l'écrit, en rapport avec les propriétés de leur langue orale, en l'occurrence le français, et les composantes de son écriture. Les écrits produits et les explications métagraphiques (dorénavant EM) qui leur sont appliquées révèlent un ensemble de stratégies "intelligentes », de procédures linguistiques et de solutions graphiques qui « approchent» les principes mêmes de l'écriture et les propriétés orthographiques qui en découlent.

L'étude s'organisera donc autour de l'analyse de plusieurs de ces productions originales ${ }^{(3)}$ afin de décrire les différents principes mis en œuvre au début de l'apprentissage, des principes qui rendent compte du processus d'acquisition de l'écriture, mais aussi de logiques générales comme la sémiographie ou la phonographie, et des procédures spécifiques appliquées par exemple à la polyvalence phonogrammique, à l'expression graphique du nombre, aux flexions et marques verbales.

L'objectif consistera à montrer en quoi les composantes de la langue, les propriétés du système d'écriture et de son orthographe historiquement construite pèsent sur les performances scripturales des jeunes élèves. Nous en déduirons des propositions didactiques conséquentes, propres à engager les enseignants à dé-

(1) Cet article adopte les rectifications orthographiques proposées dans le Journal officiel du 6.12.1990.

(2) Dans une première étude exploratoire (David, 2003c), nous avions suivi 15 élèves, âgés de 5,0 à 6,5 ans et scolarisés en grande section d'école maternelle. Par la suite, nous avons étendu notre recherche à 45 élèves du même âge et scolarisés dans les mêmes conditions (David, 2006).

(3) Le corpus de référence comprend près de 400 séquences d'explications métagraphiques transcrites, classées, puis décrites et analysées. 
ployer des apprentissages de l'écrit adaptés : ateliers d'écriture, orthographes « approchées », explicitations autographiques..., toute activité mettant les élèves dans une situation de résolutions de problèmes linguistico-graphiques, pour les aider à déployer des stratégies cognitives adaptées et des procédures ajustées. L'ensemble aura ainsi pour ambition de renouveler l'étude des écrits des jeunes élèves et les apprentissages conséquents.

Les travaux exposés ici, et ceux qui leur sont proches, ont pour visée commune d'envisager l'acquisition de l'écriture dans une dynamique générale où l'enfant interroge le monde; ce qui s'oppose à une conception de l'activité humaine réduite au simple enregistrement des données extraites de l'environnement. Nos recherches montrent en effet que les apprentis scripteurs interrogent l'écrit; ils le soumettent à leur réflexion et procèdent par tâtonnements, mais aussi par comparaison, catégorisation, mise en système des différents écrits qu'ils parviennent à produire. De fait, les jeunes scripteurs ne se contentent pas d'appréhender des formes écrites stabilisées ou normalisées ; ils les analysent, voire les reconfigurent, les redistribuent selon des principes souvent analogues à ceux décrits dans l'histoire des écritures (par exemple en utilisant le rébus ou 1'acrophonie), mais aussi en tenant compte des contraintes de la langue orale, notamment sa structure syllabique plus ou moins régulière, et les caractéristiques orthographiques du système écrit, notamment le marquage lexical et grammatical excessivement prégnant, redondant, inaudible du français.

Ainsi, les écritures enfantines que nous avons recueillies se limitent, parfois à minima, à l'inscription de simples traces et, au plus, à la composition de textes complets. Mais, quelles que soient la taille et l'envergure des écrits produits, cette pratique d'écriture « avant la lettre » ${ }^{(4)}$ est souvent identifiée sous des dénominations diverses : écritures ou orthographes inventées, approchées, essayées, tâtonnées, qui renvoient peu ou prou à l'expression anglaise : creative ou invented spelling. Il s'agit d'un paradigme de recherches, dans laquelle la profusion de travaux est en décalage avec sa relative désaffection didactique, surtout en France où la tradition normative supporte mal la production d'écrits jugés contraires à la « loi » orthographique. Nous noterons en premier lieu les travaux anglo-américains qui ont vu le jour à partir des années 1970 : Chomsky (1971, 1975), Clarke (1988), Read (1971, 1986) et se sont poursuivis au delà avec Treiman (1993), Rubin et Eberhardt (1996); mais aussi dans des études également décisives, liées à d'autres langues-écritures comme l'espagnol avec Ferreiro et Teberosky (1979), Ferreiro et Gomez-Palacio (1988), et au français avec les travaux de Besse (1990), Fijalkow et Fijalkow (1991), Jaffré (1992), David et Jaffré (1997), David (2003a, 2003b), Morin (2004, 2005), Morin et Montésinos-Gelet (2006)... Le projet de cet ensemble de recherches ${ }^{(5)}$ est d'étudier les productions écrites de jeunes enfants, avant les apprentissages réguliers et systématiques de la lecture-écriture, et de décrire le processus d'émergence d'une litéracie en suivant des protocoles et des méthodologies qui peuvent varier : de la simple production écrite sans révision (essentiellement dans la tradition anglosaxonne), à la relecture-réécriture normée par des échanges entre pairs, et avec l'enseignant, selon une démarche plus proche des travaux francophones : Jaffré et Ducard, 1996 ; David, 2006 ; David et Morin, 2008.

(4) Pour reprendre la très belle expression que Ferreiro donna à l'une de ses premières études : «L'écriture avant la lettre », dans Sinclair (dir.), La Production de notations chez le jeune enfant, Paris, PUF, 1988.

(5) Pour une synthèse argumentée de ces différents travaux et du courant de recherches qui les constitue, voir Jaffré et al. (1999). 


\section{Approcher les propriétés de l'écriture}

\subsection{La sémiographie : signifier graphiquement}

Nous pouvons affirmer aujourd'hui que les élèves n'entrent pas dans l'écrit à une date ou une période déterminée. En fait, l'écriture de leur langue, par ses propriétés, ses caractéristiques, ses contraintes... s'impose à eux et transparait de façon plus ou moins homogène dans leurs premiers essais d'écriture. Ainsi, dans une étude longitudinale de cinq très jeunes enfants, nous avons montré que les premiers tracés, recueillis dès 1,6 ans, montrent qu'ils pratiquent précocement l'écriture, et n'y entrent pas à un moment déterminé. De fait, des expressions comme «l'entrée dans l'écrit » (Fijalkow, 1993)... à cinq ou à six ans se réfèrent en fait aux apprentissages scolaires ou, comme l'énoncent Besse et al. (1999), à « l'entrée dans la phonétisation de l'écriture ». Dans le cadre de telles études, si les élèves 《 entrent» dans l'écrit, cette " entrée » correspond plus au début d'un enseignement programmé et non aux activités scripturales spontanées, ou induites, que nous avons observées en amont. Les très jeunes scripteurs de notre étude sont ainsi immédiatement confrontés aux formes écrites, qu'ils les voient, qu'ils les interrogent ou qu'ils se les approprient dans une démarche qui emprunte, certes, plus à l'imitation qu'à une production originale, tout du moins dans un premier temps.

Les tracés ou proto-écritures recueillis ${ }^{(6)}$ montrent ainsi des tendances liées à la découverte des instruments scripteurs, à la reproduction de gestes et de formes caractéristiques de 1'écrit : cycloïdes, traits continus ou discontinus, lignes droites ou brisées, boucles... inscrits dans toutes les directions offertes par l'espace plan. La maitrise de ces tracés n'est guère contrôlée ; ils semblent inscrits sans intention signifiante et visent plutôt à remplir l'espace graphique :

Fig. 1 : Laure à 1 an 10 mois (Proto-écriture avec stylo fin noir sur enveloppe kraft)

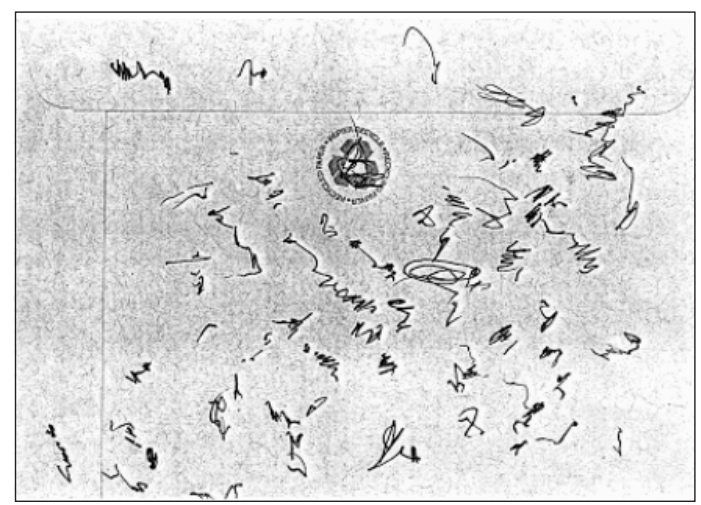

Les outils d'écriture et les supports apparaissent en revanche très tôt conventionnels; ils correspondent à ceux qui sont privilégiés dans les pratiques scripturales ordinaires (enveloppe, page lignée, post-it...).

Par la suite, l'imitation des gestes s'affirme pour laisser apparaitre des graphismes et parfois des segments dont la spatialisation devient progressivement plus conventionnelle (de gauche à droite) et en lignes distribuées de haut en bas. Ces graphismes peuvent parfois faire « signe » et fonctionner comme des « mots », mais exclusivement lorsqu'il s'agit de noms propres. Nous suggérons que ces tracés préfigurent les futurs sémiogrammes, massivement recueillis dans les études autographiques. L'attention aux tracés est alors plus contrôlée. Les enfants reproduisent ou non les formes conventionnelles de l'écrit, et leurs gestes correspondent à une réelle

(6) Pour ce faire, nous avons constitué un corpus de près de 1000 figures sur quatre ans pour cinq enfants suivis en crèche puis en petite et moyenne section d'école maternelle. 
imitation de l'écriture adulte. Les supports sont toujours aussi caractéristiques des usages normés de l'écriture, avec des choix d'instruments correspondant encore plus à l'activité scripturale adulte (stylo fin ou à bille, stylo plume...).

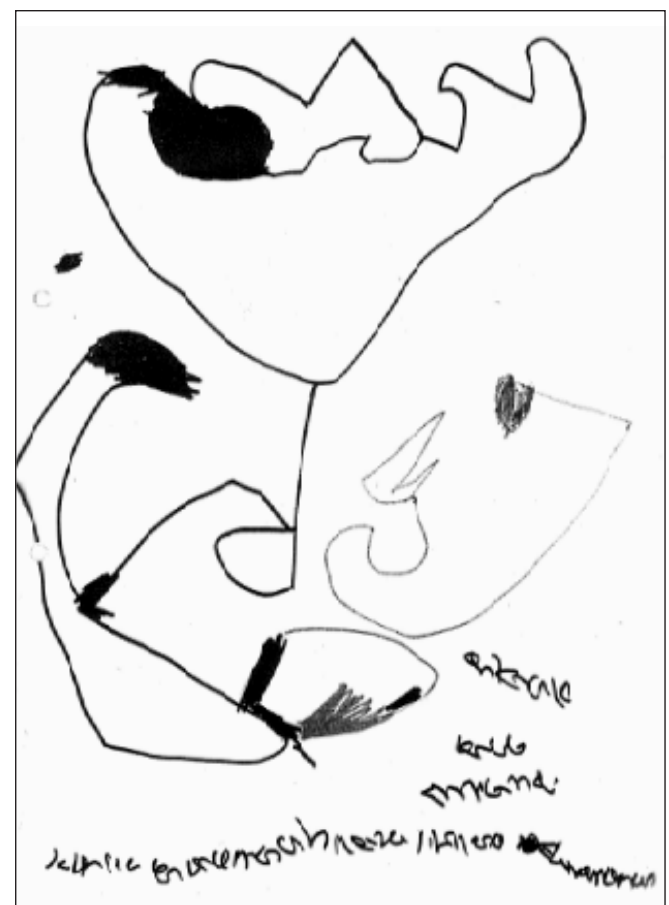

Les mois qui suivent, les graphismes apparaissent en nombre croissant. On peut même évoquer une véritable explosion de la proto-écriture au tournant de la troisième année. Il est vrai que la plupart des enfants entrent à l'école maternelle où la perception des traces écrites y est accrue : manipulations d'étiquettes diverses dont les prénoms, repérages sur des calendriers, des listes de tâches, ateliers de graphisme. Les dessins apparaissent également mais avec un décalage certain avec la proto-écriture (fig. 2 ci-contre).

Fig. 2 : Laure à 3 ans 5 mois (dessin de fleur + proto-écriture avec feutre fin noir sur feuille blanche)

Comme en atteste les commentaires appliqués à cette double production, iconique et scripturale, de Laure :

\section{Laure (3,5 ans) - PS d'école maternelle EM pour l'ensemble}

Lau (montre le dessin) : c'est une fleur -j'ai pas fini de colorier

Ad : elle est très jolie - tu la termineras tout à l'heure - et là c'est quoi tout ce que tu as fait là (montre les graphismes inscrits sous la fleur)

Lau : c'est des noms

Ad : ah oui et quels noms

Lau : j'ai écrit Laure et pi Lucille et Aurore et là (montre la dernière ligne comportant plusieurs segments) - "une fleur pour Lucille".

Par la suite, la démarche signifiante apparait pour les protosignes mais elle reste rétroactive ; à l'inverse du dessin qui semble désormais liée à une intention picturale. De fait, l'interprétation en termes de proto-écriture est appliquée aux traces et non l'inverse (voir fig. 3 page suivante) :

\section{Laure $(4,4$ ans) - MS d'école maternelle}

\section{EM pour l'ensemble (dessin et proto-écriture)}

Lau (commente son dessin :) c'est un garçon avec des fleurs et une maison-j'ai fait des nuages aussi mais je sais pas encore bien les faire

Ad : c'est très joli-tu as mis du rose pour les fleurs - et là c'est quoi (montre les suites de lettres)

Lau : j'ai écrit le nom du garçon

Ad : et tu as écrit quoi 
Fig. 3 : Laure à 4 ans

4 mois (dessin + protoécriture stylo à bille noir sur feuille blanche)

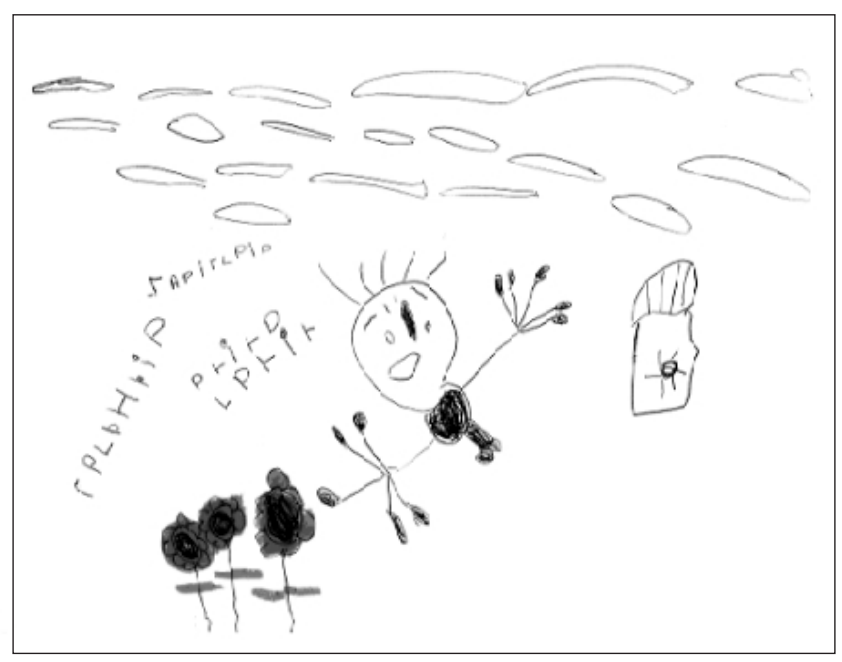

Lau : j'ai écrit -j'ai écrit heu - Nabil

Ad : et ici aussi c'est Nabil

Lau (pointe successivement les deux segments proches du garçon :) nonj'ai écrit - -j'ai écrit qu'il rêve qu'il a deux noms

Comme on le voit, les commentaires restent fortement induits par le questionnement adulte qui invite - ou précipite parfois - les réponses en termes de mots ou d'énoncés. Les échanges montrent ainsi que les enfants distinguent parfaitement l'activité scripturale de l'activité figurative. Les « mots » ou sémiogrammes sont énoncés sans déterminant ; ils sont montrés et parfois réinterprétés dans des commentaires qui varient en fonction des compétences discursives des sujets. Les tracés sont plus assurés et suivent des trajectoires désormais plus normées : de gauche à droite et de haut en bas. Les graphismes se diversifient également tout en se spécifiant du côté de l'écriture. De toute évidence, ces segments se rapprochent des séries de mots ou de lettres observées sinon imitées de l'environnement scriptural immédiat.

L'activité de proto-écriture est toujours intensive jusqu'à la fin de la moyenne section (5 ans environ). Les graphismes associent de plus en plus de lettres conventionnelles et correspondent parfois à des mots copiés ou reproduits de mémoire. Pour certains enfants, ces proto-écritures spontanées disparaissent complètement à partir du moment où les lettres et les mots normés sont découverts et définitivement incorporés aux «écrits » produits. Mais, pour la plupart, des lettres et des pseudo-lettres sont mêlées dans des productions en nombre toujours plus important. Pour l'ensemble de ces jeunes scripteurs, la démarche est désormais socialisée ; elle combine l'écriture et la « lecture » des éléments inscrits : les enfants demandent aux adultes de décoder ce qu'ils ont inscrit. L'activité de copie - reproduction de modèles pourtant pratiquée de façon insistante en moyenne et grande sections - reste marginale, limitée au prénom de l'enfant et liée pour l'essentiel aux exercices scolaires ; de fait, elle commence à être utilisée dans les proto-écritures ou écritures spontanées. Notons que parfois, à l'instar de Laure, les dessins sont associés aux protosignes dans le même espace, alors que la majorité des enfants préfèrent les distinguer des productions analogiques. Les segments sont interprétés plus systématiquement; ce sont des signes, des lettres ou des nombres qui sont nommés : «c'est $O »$, «c'est $2 » \ldots$ et le plus souvent des noms, au pre- 
mier rang desquels les prénoms : «j'ai écrit Nabil », «c'est presque mon nom... Céline ». Pour Laure, les mots ainsi déduits des segments de signes ou protosignes sont parfois compris dans des amorces ou des micro-récits qui accroissent la valeur discursive de leurs proto-écritures.

Au delà, pour tous les enfants suivis, les gestes graphiques s'affinent au fur et à mesure que les formes normées (lettres, mots) apparaissent. Cependant, les lettres capitales sont privilégiées, car elles présentent une plus grande facilité d'exécution, même s'il n'est pas rare de constater quelques tentatives d'écriture cursive, mais pour les prénoms uniquement.

Cependant, si l'imitation est d'abord et essentiellement celle des activités adultes observées dans la fratrie ou auprès des pairs, on note que c'est vers 4 ans et demi (pour les premières tentatives) que les enfants reproduisent délibérément des lettres et des mots normés mis à leur disposition. Les compétences de copie sont donc à la fois largement induites par l'enseignement, et distinctes des protoécritures spontanées. De même, nous montrons que l'effort de signifiance part toujours du graphisme, des protosignes inventés ou des lettres et mots empruntés à des écrits présents dans les différents milieux : famille, crèche et école. On peut même dire que le signifié se (re)construit autour de ces protosignes pour être reconnus en tant que signifiant graphique. De fait, pour la plupart des sujets de notre étude, ces capacités de signifiance apparaissent indépendamment des performances graphiques.

L'interprétation des protosignes (graphismes non conventionnels ou pseudolettres) apparait dès 4 ans 4 mois, c'est-à-dire à un âge où la maitrise des gestes et des tracés est loin d'être accomplie. Il n'y a donc pas de rapport direct entre la qualité du graphisme - ou sa valeur conventionnelle - et la reconnaissance de signifiants écrits, pour l'essentiel des noms propres (Nabil, Aurore...). Cette nouvelle perception de l'écriture comme ensemble de signifiants - ou protosignes signes - marque un tournant décisif de la conceptualisation de l'écrit; nous qualifions cette démarche de sémiographique ${ }^{(7)}$.

Dans une autre perspective, nous observons que les deux ordres de l'oral et du scriptural présentent des différences et des similarités en termes de développement; ils ne sont donc pas opposés (Peytard, 1970) dans 1'approche ontogénétique qui est la nôtre. Les dissemblances peuvent être établies au plan des procédures psycholinguistiques, puisque les mots écrits trouvent leur origine dans les protosignes décrits ; ils ne se déduisent pas de leur équivalent oral. On peut même affirmer que l'encodage n'est pas une démarche que les enfants mettent en œuvre spontanément dans leur protoécriture ; 1'écrit préexiste dans un processus d'interprétation et non de transcription des unités de l'oral, qu'il s'agisse de lexèmes identifiés ou de sons plus ou moins largement extraits. Le rapprochement des deux ordres, de l'oral et du scriptural, apparait en revanche dans les stratégies d'acquisition, puisque les protosignes de l'écrit acquièrent progressivement un statut de signifiant dans une médiation explicite. De façon similaire, les mots de l'oral nécessitent une médiation de même nature pour être reconnus, intégrés et reproduits dans les discours oraux des enfants. Le sens à l'oral comme à l'écrit découle d'une activité signifiante guidée par un locuteur ou un scripteur expert et dépend ainsi en grande partie d'un « dialogisme contrôlé » (François et al., 1984).

(7) Nous préférons le terme de sémiographie à celui de logographie qui correspond généralement, dans les travaux sur l'acquisition de la lecture - et plus globalement de la litéracie -, à une phase plus ou moins délimitée dans les modèles à étapes ou "étapistes ». 
L'étude de ces différentes stratégies sémiographiques nous semble déterminante parce qu'elles correspondent à une logique d'écriture primordiale dans les deux perspectives : ontogénétique et phylogénétique. Sur ce plan, nous montrons que ces démarches accélèrent la mise en place de compétences scripturales qui vont gagner en intentionnalité et en conventionnalité, par le fait des apprentissages induits par l'école, et parfois par l'environnement familial. Certes, nous constatons que, dans cette sémiographie émergente, les jeunes scripteurs ne raisonnent pas encore sur les propriétés phonographiques ou alphabétques de l'écrit; ils sont cependant confortés dans leurs démarches par l'adéquation de ces traces sémiographiques aux normes de l'écrit, comme dans la production désormais plus normée de Jamila :

Fig 4 : Jamila à 5 ans 6 mois (première version d'un texte en gestation)

\section{dovidmarian
piscine}

Jamila énonce ainsi un récit minimum, certes réduit aux lexèmes les plus saillants : les personnages (david marion) et un élément du procès (piscine), qu'elle commente ainsi :

Jamila (5,6 ans) - GS d'école maternelle

Texte écrit en mai : david marion/piscine (= David et Marion vont à la piscine) EM pour le « et » manquant :

Ad : comment tu as fait pour écrire ces mots

Jam : et ben david et marion je sais déjà l'écrire - on l'a déjà mis ici (montre une affiche)

Ad : et pour piscine

Jam : je l'ai copié du livre de la classe

$\mathrm{Ad}$ : tout à l'heure tu m'as bien lu ton histoire - tu peux me la relire et me montrer où tu as écrit et

Jam : (relit en pointant chaque mot) David-- Marion--j'ai oublié il faut le mettre là (indique un espace entre les deux prénoms).

Cet exemple témoigne parfaitement de la mise en œuvre d'une démarche sémiographique plus conventionnelle ou «légitime ». Les mots existent dans l'environnement. Les procédés utilisés renvoient soit à des mots mémorisés holographiquement, soit à des démarches de transport-copie, parfaitement décrites par ailleurs (Rieben et Saada-Robert, 1997). Au cours de cette activité, l'écriture est légitimée ou socialisée, parce que potentiellement lisible, avant d'être conceptualisée dans un apprentissage linguistique. Pourtant, dans cette approche " globalisante » de l'écriture, on ne peut encore parler de véritable acquisition systémique ; sauf à envisager que chaque enfant doive produire un effort singulier pour chacun des mots qu'il voudra inscrire ou reconnaitre. Pour que l'acquisition débute vraiment, il faut qu'émergent des hypothèses "intelligentes » du fonctionnement de l'écriture, et notamment la formulation de principes alphabétiques et morphologiques tour à tour spécifiques et généraux.

\subsection{La phonographie : saisir l'économie du système écrit}

La démarche phonographique décrite dans nos travaux - et d'autres inscrits dans le paradigme des orthographes « inventées » (supra) - constituent un mode de repré- 
sentation qui peut soit compléter soit déranger les procédures sémiographiques précédemment décrites. Il ne s'agit plus de représenter directement du sens par des mots mais de saisir les sons de la langue pour les représenter par d'autres graphies, même si ce sont les mêmes sémiographies sensiblement reconfigurées.

Ces écrits inscrits par l'application de diverses procédures phonographiques sont déterminantes pour l'analyse des mécanismes cognitifs à l'œuvre dans les apprentissages de l'écriture. Nous considérons en effet qu'elles sont les traces visibles, généralement motivées et non conventionnelles, de procédures acquises ou en cours d'acquisition. Nous les étudions donc non par rapport à un système orthographique normé et plus ou moins maitrisé, mais par rapport aux démarches accessibles ou effectivement mises en œuvre par ces jeunes élèves. De ce fait, nous ne pouvons les appréhender en fonction de critères linguistiques externes, et conformes aux attentes scolaires ou sociales; nous devons, au contraire, les évaluer selon des critères d'évolution internes, comme des démarches "intelligentes »observées dans le cours même de ces écritures autographes. Nous avons ainsi régulièrement constaté, avec ces jeunes scripteurs, que l'évolution de leurs stratégies pouvait déstabiliser leur entourage ; notamment lorsqu'ils optent pour cette démarche phonographique (par exemple en écrivant mézon, alors que, quelques jours auparavant, ils l'écrivaient de façon normée, maison, en apparence plus légitime et conventionnelle). Ce changement de logique semble déboucher sur une perte de qualité - ou plutôt de "normalité ». En fait nous montrons qu'il s'agit d'un gain procédural considérable, comme pour Lorie qui écrit puis explique :

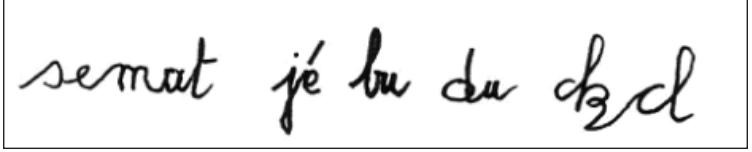

Fig 5 : Lorie à 5 ans 11 mois (première version d'un texte en cours de réécriture)

\section{Lorie (5,11 ans) - GS d'école maternelle}

\section{Récit de vie écrit en juin : Ce matin j'ai bu du coca-cola}

EM pour semat (= ce matin)

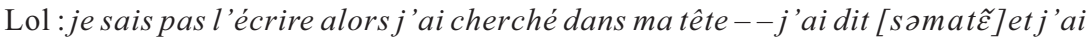
trouvé ces lettres là.

EM pour $c k c l$ (= coca-cola)

Lol : j'ai mis quatre lettres parce que ça fait quatre lettres dans [ko-ka-ko-la] et pi [ka] je sais l'écrire c'est comme au début de Karine - c'est ma copine.

Lorie utilise ainsi des procédures phonographiques fonctionnelles, en extrayant les unités perceptibles dans l'énoncé oral choisi. Elle explique parfaitement que, confrontée à l'impossibilité d'une notation idéovisuelle des mots ( $j e$ sais pas l'écrire...»), elle recourre à une démarche empirique ("... alors j'ai cherché dans ma tête ») qui combinent diverses procédures phonographiques : un codage partiel des phonèmes les plus saillants révélant une connaissance déjà ajustée des graphèmes réguliers ( "j'ai dit [samat $\tilde{\varepsilon}]$ et j 'ai trouvé ces lettres là »), puis une transcription syllabique ( " j'ai mis quatre lettres parce que ça fait quatre lettres dans [ko-ka-ko-la] ») qui s'appuie sur des savoirs construit relativement à la valeur épellative des lettres (" [ka] je sais l'écrire c'est comme au début de Karine »).

Nous montrons ainsi que les procédures alphabétiques tâtonnantes de ces jeunes élèves révèlent des procédures linguistico-graphiques approchant l'économie du système alphabétique. Elles apparaissent inévitablement non-normées, mais 
fondamentalement évolutives, car elles sont comprises dans des apprentissages progressifs et dynamiques. À la lumière de ces descriptions, nous considérons ainsi que, pour apprendre à écrire, il faut certes que les enfants soient en contact avec les formes conventionnelles de l'écriture, mais il faut surtout qu'ils les confrontent avec leurs représentations empiriques et les principes sous-jacents à l'écriture de leur langue, et sans doute avec les logiques phonographiques à l'œuvre de façon variable dans tous les système d'écriture (Jaffré et David, 1999).

Ajoutons que ces écritures approchées conduisent nos jeunes scripteurs à explorer un autre univers langagier, un univers accessible par le déploiement d'une activité nouvelle, spécifique et complexe, une activité essentiellement métalinguistique parce qu'intrinsèquement appliquée aux objets écrits. Alors qu'ils maitrisent l'essentiel des bases du langage oral - acquises de façon implicite -, sans recourir ni à des explications, ni à une technologie grammaticale ou lexicale, ces jeunes scripteurs doivent dorénavant, et parce qu'ils écrivent, questionner des formes orales et écrites, trouver des solutions graphiques, appréhender les structures et composantes du système écrit, déployer de nouvelles logiques linguistiques ; bref produire des raisonnements appliqués à l'écriture de cette langue qu'ils pratiquent de façon implicite. C'est assurément une expérience à la fois nouvelle et originale, une expérience qui transforme leur rapport au monde et aux autres, mais aussi, et de façon spécifique, une expérience qui change leurs fonctionnements cognitifs et leurs savoirs sur la langue. Dans ce sens, nous constatons que le recours à la phonographie constitue un passage décisif dans la genèse de l'écriture des jeunes enfants. Ce passage peut se résumer par les caractéristiques suivantes:

— Un rapport à la langue et au signe transformé, car dans la plupart des écrits produits et dans les EM qui les suivent, les enfants doivent considérer les mots non comme des signes-sens qui serviraient directement à « dire » le monde (à l'aide de mots-étiquettes), mais comme des unités linguistiques qu'il faut transcrire selon une logique singulièrement opposée. Dans ce mouvement, le signe acquiert alors un statut réellement linguistique, dans le sens où il est encore plus démotivé et distinct du signifié. Il est reconnu avec une fonction désormais arbitraire et définitivement abstraite du réel. En celà les signifiants écrits ne notent plus directement les choses ; ils les codent indirectement via la structure sonore de la langue. C'est ce que découvrent les élèves de notre étude lorsqu'ils s'essayent à transcrire les unités de l'oral. Réciproquement les signes écrits, principalement les lettres, sont reconnus et utilisés dans de nouvelles fonctionnalités ; ils sont la source de raisonnements et d'opérations parfois originales (Bissex, 1980 ; Jaffré, 1992), des opérations que nous pouvons le plus souvent décrire dans la révision de leurs écrits.

- La nécessité de tout écrire, car le passage à la phonographie est souvent lié à une prise de conscience nouvelle : celle de noter toutes les unités perçues dans le message oral, y compris celles qui ne sont pas porteuses d'un sens plein comme les substantifs. Il s'agit ainsi de noter également les verbes et parfois leurs flexions, les adjectifs, mais aussi et surtout les mots grammaticaux (dans l'ordre de fréquence : les déterminants, les pronoms et les prépositions). De fait, ces termes n'ont pas de raison d'apparaitre dans une logique sémiographique, comme nous l'avons vu précédemment; ils sont en revanche perçus et nécessairement transcrits lorsque les enfants adoptent une démarche phonographique.

- L'apparition de nouvelles unités graphiques, car ce ne sont plus les mots qui sont privilégiés dans la composition des textes, mais les sons et les lettres qui leur correspondent plus ou moins. En employant les termes de sons et de letres, nous avons conscience de leur approximation, puisqu'il s'agit d'un ensemble d'unités plus complexes et à consistance variable qui vont : du phonème à la syllabe 
(comme dans le texte et les EM de Lorie ci-dessus) en passant par ses attaques ou ses rimes (codage analogico-alphabétique et épellatif: «L $m f$ des Kane» (= « elle me fait des câlins »). Ces démarches d'encodage sont tâtonnantes et sources d'《erreurs » particulières. Elles aboutissent à des solutions graphiques généralement partielles : une lettre pour un mot court, généralement monosyllabique («je vous $m$ tous », avec pour EM : «j' ai mis $M$ ça fait aime»), ou pour une syllabe dans des mots plurisyllabiques (« un koe » (= un cadeau) et l'EM suivante : « j'ai dit [ka] c'est dans Karine la lettre en premier et puis après [do] j'ai écrit le $O »)$ ou pour une partie de la syllabe, à l'attaque («jn bi», commenté ainsi : "C'est un petit mot quand on dit j'aime on entend [3::] en premier ») ou à la rime ( $p r$ Noël» (= Père Noël) », justifié par : "c'est père - j'ai entendu un $P$ et un $R$ - on l'avait écrit mais j'ai pas regardé j'ai dit le mot dans ma tête »), le plus souvent, une suite de lettres plus ou moins en adéquation avec les phonèmes extraits dans un mot ( «ous pouvé ", parce que «ça s'écrit comme ça pouvé ») et parfois une suite de mots ( «on vavite » parce que j'ai dit le mot et j'ai pensé à ces lettres là un $V$ un $A$ un $V$ un $I$ un $T$ un $E$ ça fait va vite »).

- L'émergence de conflits entre des conceptualisations opposées, car le principe phonographique peut gêner une perception visuographique. C'est le cas de la notation syllabique de mots courts où la quantité d'unités inscrites - inévitablement réduite à une lettre - s'oppose à l'idée enfantine qu'un mot doit en comporter plusieurs (voir également Ferreiro \& Gomez-Palacio, ibid.). Ce conflit est plus ou moins résolu, ou plutôt contourné, par l'ajout de lettres aléatoires, par exemple un $o(=\mathrm{au})$ complété par deux lettres, le, " qu'on (n')entend pas », ou encore un $e$ qu'« il y a des fois à la fin des mots »et donc ajouté à koe (= cadeau). De fait, le recours à des procédures phonographiques apparaissent plus rationnelles, plus « économiques » ou « systémiques », dans une logique dorénavant linguistique ; mais en même temps elles perdent le caractère conventionnel ou «légitime» des mots copiés précédemment selon une logique plus sémiographique.

- Une autre distribution des procédures liée aux catégories linguistiques, car la phonographie ne se déploie pas de façon uniforme et indistincte. Les mots courts, généralement monosyllabiques, sont souvent codés par l'application de procédures phonographiques, qu'il s'agisse de leurs phonèmes, de leurs syllabes ou des attaques/rimes de ces syllabes, alors que les mots longs le sont par le recours à des mots copiés ou mémorisés idéovisuellement. Il arrive cependant que certains élèves optent pour ces procédures phonographiques quelle que soit la nature des mots à inscrire; ce sont alors des phonèmes ou des syllabes de substantifs plus ou moins complets qui sont ainsi codés. Il reste que les mots grammaticaux (déterminants, pronoms, prépositions...), parce qu'ils sont généralement monosyllabiques, sont transcrits selon des procédures plutôt syllabiques ou épellatives. De fait, les tentatives de notation alphabétique s'appliquent plus à des formes partielles (les trois-quarts des procédures recensées) qu'à des formes complètes (seulement un quart des procédures observées).

Il reste que la phonographie ne transforme pas radicalement ou définitivement les autres conceptions et procédures d'écriture. Elle semble au contraire s'appuyer sur les connaissances sémiographiques antérieures ou parallèles; elle les assimile, les reconfigure dans une logique qui reste, à terme, toujours l'expression du sens. De fait, nous constatons que les élèves de notre étude ne se contentent pas de reproduire des formes écrites, de les copier ou de les recopier, ils se les approprient en les interrogeant. Pour ce faire, ils n'hésitent pas à déployer des procédures qui relèvent à la fois de l'inventivité graphique, de l'ajustement aux formes 
écrites normées et de 1'exploration des principes disponibles dans le système d'écriture de la langue. Ainsi :

- L' « inventivité graphique » est manifeste dans des tentatives graphiques qui apparaissent originales, mais qui sont tout à fait plausibles, comme le repérage des syllabes et leur notation (codage syllabico-alphabétique : «C'est poupée - j'ai mis un $P$ et un $E$ on l'entend [pu-pe]»), ou encore la dénomination des lettres et leur inscription (codage épellatif : " il faut un $M$ on entend [ $\varepsilon \mathrm{m}]$ dans $t$ 'aime »). Ces deux procédures existent dans des systèmes autres que le français écrit : par exemple les syllabaires sont à la base du système des Kana japonais ; ou l'épellation est utilisée pour coder les noms propres en serbocroate. La première marque également le passage historique des écritures idéographiques aux écritures alphabétiques. Cette inventivité phonographique apparait ainsi comme le moteur de l'activité graphique; les jeunes scripteurs l'explorent de façon parfois intensive. Ils montrent ainsi que le cerveau humain n'est pas une chambre d'enregistrement des stimulus graphiques extérieurs, ou même d'une machine pré-programmée qui actualiseraient les règles et les formes linguistiques. Comme dans la phylogenèse, l'ontogenèse de l'écriture se caractérise par des développements endogènes et exogènes, et par la construction de théories linguistico-graphiques tour à tour provisoires et stabilisées ${ }^{(8)}$. Les élèves de notre étude se comportent en fait comme des «apprentis linguistes » qui questionnent les formes écrites, interrogent les fonctionnalités du système et les assimilent également dans des conceptualisations dynamiques, souvent provisoires, parfois stabilisées.

- L'ajustement aux formes écrites conventionnelles est patente lorsque les jeunes scripteurs révisent leurs textes. Nous avons montré que, dans les échanges avec les pairs ou avec l'adulte, les solutions phonographiques sont souvent reconsidérées à la lumière d'arguments sémiographiques. La démarche va dans le même sens quand ils généralisent les principes de transcription à partir d'analogies phonographiques (comme dans cette EM : «ça fait [1ə] je le connais - dans le vélo »). Ces ajustements sont indispensables car les élèves ont conscience que leurs solutions graphiques ne correspondent pas à l'orthographe normée ; ils vérifient ou corrigent donc leurs productions en recourant à des mots présents dans un environnement plus ou moins proches. Ils associent ainsi deux logiques d'écriture : un codage indirect et une notation directe des signifiés. Ces deux logiques sont loin d'être irréductibles l'une à l'autre. Nous considérons qu'elles sont complémentaires, faute de quoi une part importante de l'orthographe lexicale et grammaticale du français (les lettres muettes, les consonnes doublées, les marques morphographiques ou idéographiques...) échapperait à tout apprentissage.

— L'exploration des principes disponibles dans l'écriture de leur langue est récurrente car ces enfants saisissent rapidement les régularités alphabétiques, des voyelles (comme Latifa : "J'ai mis un $A$ - on entend [a] ») ou des consonnes (comme Nabila : "Quand on dit j'aime on entend [3::] en premier»). Mais surtout, ils manifestent très tôt une sensibilité aux irrégularités phonogrammiques, et notamment à la polyvalence graphémique, particulièrement importante dans les flexions verbales (comme chez Benjamin : «parce que à la fin c'est [e] avec $E-R$ - on peut l'écrire avec le $E$ de février - non le $E$ - $R$ à la fin - je préfère pouver comme ça »), même si les solutions choisies à terme ne sont pas toujours conformes. Cette sensibilité à la variation graphique est décisive pour comprendre et, ultérieurement, maitriser les fonctionnements orthographiques du français. Nous

(8) Dans cette perspective, voir Karmiloff-Smith (1992); Gopnick et Meltzoff (1997). 
voyons ainsi que les premières tentatives d'écriture alphabétique ne se limitent pas à l'application mécanique de règles de correspondances phonogrammiques ; ces règles sont spécifiées, ajustées, reconfigurées dans des raisonnements qui tiennent compte autant des possibilités du système que de ses contraintes. Cette capacité d'ajustement des règles en fonction des réalités linguistiques est désormais bien étudiée et analysée dans des modèles d'acquisition du langage oral (voir notamment Bybee, 2001) et écrit (Rittle-Johnson et Siegler, 1999). Nous nous y référons également pour montrer que l'apprentissage de l'écriture ne résulte pas de l'application d'un principe monolithique, mais bien de l'ajustement des différentes procédures psycholinguistiques aux réalités linguistiques.

D'autres recherches ont inventorié et analysé ces procédures phonographiques chez des enfants de 5 à 6 ans, scolarisés ou non. Les plus étendues sont sans conteste celles de Ferreiro et coll. (de 1979 à 1988), déjà signalées. Cependant, la méthodologie, sensiblement différente de la nôtre, ainsi que les contextes et caractéristiques de la population ${ }^{(9)}$ étudiée ne peuvent nous conduire vers des comparaisons terme à terme. Il reste que Ferreiro et Gomez-Palacio (ibid.) ont proposé un ordonnancement des conceptualisations du lire-écrire qui obéit en grande partie à des principes d'évolution interne, selon un modèle constructiviste. Ce modèle en étapes, niveaux ou paliers n'est pas vraiment le nôtre. Nous avons montré que loin de recourir successivement à des procédures hiérarchiquement ou chronologiquement organisées, les élèves francophones de notre étude utilisent conjointement - et souvent complémentairement - des procédures supposées inconciliables. Ainsi, nous avons déjà montré qu'ils suivent des logiques d'écriture parfois antagoniques, qu'ils combinent l'usage de logogrammes et de phonogrammes, qu'ils recourent à des procédures phonographiques hétérogènes. Ainsi, à l'intérieur de ce champ phonographique, ils associent différentes procédures : phonogrammiques, analogiques, syllabiques, épellatives, dans l'inscription d'un même texte - et donc dans le même temps d'écriture - et souvent dans le codage d'un même mot ou la révision d'une même unité. D'autres travaux complémentaires des nôtres dans le domaine de la lecture (Goswami, 1988) et de l'écriture (Seymour, 1993, 1997 ; Treiman et Bourassa, 2000 ; Morin, 2004a, 2004b), suggèrent également une conception «multidimensionnelle » du développement de l'écrit, et notamment la capacité des apprentis lecteurs-scripteurs à utiliser précocement - et sans les opposer - les informations sémio-, phono- et morphologiques.

Dans le domaine phonographique, Ferreiro et Teberosky (ibid.), puis avec Gomez-Palacio ( $i b i d$.), ont également formulé une hypothèse qualifiée de « syllabique ", hypothèse qui suggère que les enfants prélecteurs (et apprentis scripteurs) passe par une phase de notation des syllabes de façon quasi obligée. En fait, ce passage ne nous semble pas systématique ; la plupart des élèves de notre étude n'y recourent pas, d'autres l'utilisent mais de façon marginale. Nous montrons ainsi que le codage syllabique, s'il est effectif pour certains élèves, n'apparait ni majoritairement ni massivement à une période déterminée de 1'apprentissage. Que faut-il en conclure ? Si la syllabe constitue, certes, une source rentable du codage phonographique, elle n'est cependant pas la seule. De fait, nos jeunes scripteurs semblent privilégier d'autres unités (les phonèmes et les lettres) sans doute parce que

(9) Rappelons que, dans leurs études, Ferreiro et ses collaborateurs ont privilégié des protocoles expérimentaux, contrôlés en amont sur les plans linguistique et cognitif: production de lettres et de chiffres, écriture et analyse de prénoms, analyse des parties de phrases, permutation de phrases, écriture de mots et de phrases, écriture avec images, lecture avec images ; avec en plus le projet de ne pas scinder les processus de lecture et ceux d'écriture. 
la consistance de la syllabe en français, plus qu'en espagnol, n'offre pas les mêmes possibilités ni les même fonctionnalités. D'autres phénomènes concourent à la saillance ou, au contraire, à l'amuïssement de la syllabe. De récents travaux en phonologie, incluant les faits de prosodie (Angoujard, 1997), montrent que le rythme, les courbes mélodiques... et surtout l'accentuation de mot, prégnante en espagnol et quasiment absente en français, modifient considérablement la perception des syllabes et des phonèmes qui les composent. Les voyelles espagnoles sont mieux perçues que les voyelles françaises et plus encore que celles de 1'anglais (Treiman, 1989 ; Treiman et al., 2002). Les consonnes, en revanche, sont mieux discriminées au plan graphique et porteuses d'un maximum d'information visuelle, en français et en anglais ${ }^{(10)}$. Il semble donc que $l^{\prime}$ « hypothèse syllabique » envisagée par Ferreiro, et d'autres à sa suite, doive être revue à la lumière de descriptions contrastives et linguistiquement plus fines. De fait, nos jeunes scripteurs ne perçoivent pas de façon univoque les différentes unités de leur langue orale ${ }^{(11)}$. La structure des syllabes, mais aussi leur consistance phonologique et leurs contextes d'emploi déterminent en grande partie la capacité de ces mêmes scripteurs à inscrire les mots et à composer leurs textes. La structure graphique des mots agit également sur la manière de privilégier tantôt les lettres tantôt les sons. Certains systèmes d'écriture, notamment ceux dont la structure consonantique est prégnante (voir Ravid, 2001, 2006, pour l'hébreu), déterminent les productions orthographiques des élèves. C'est parce que les écritures sémitiques n'inscrivent que les consonnes (au détriment des voyelles, peu informatives car moins nombreuses) qu'elles amènent les apprentis scripteurs à ne noter que ces consonnes (voir également Teberosky et Tolchinsky-Landsmann et al., 1993). On peut donc limiter la portée de cette «hypothèse syllabique » en affirmant qu'elle est liée avant tout à la qualité (régularité et saillance) phonographique de cette unité en espagnol, qu'il s'agisse du castillan ou du catalan.

Dans une perspective également psycholinguistique, Jaffré (1992) a proposé une typologie hiérarchisée des procédures graphiques en les extrayant d'un large corpus d'écrits et de commentaires produits par des élèves du même âge. Il détaille ainsi les différentes procédures phonographiques fonctionnelles observées dans son corpus :

- épellatives où les lettres sont employées pour leur dénomination (ex : 《 gâteau » / K ; « aider» / D) ;

- syllabiques où les lettres représentent des syllabes (ex : « farine »/ JAI ; « Paris »/PR);

- logo-syllabiques avec ajout de lettres aux lettres-syllabes (ex : « farine »/ AIEUA) ;

- phonogrammiques où les lettres représentent certains phonèmes d'un mot (ex : « poivre »/ PAVR ; « cidre »/ SIRE) ;

- logo-phonogrammiques ou squelettes dont les lettres représentent tous les phonèmes d'un mot (ex : « affiche »/ AFIH ; « cheveux » / CHEVE);

- distributionnelles avec un choix des lettres déterminé par l'environnement graphique (ex : « couleur» / COULER vs « sur»/ CUR)

(10) Ce phénomène de saillance visuographique des consonnes est à relativiser dans certains contextes : dans les groupes consonantiques ou clusters en attaque de syllabe ou en fermeture de rime, et surtout en fin de mot.

(11) Nous renvoyons à l'exposé de ces contraintes orthographiques spécifiques du français dans les travaux de Catach (1995), des contraintes qui déterminent sensiblement les différentes pratiques de l'écriture, ordinaires ou savantes, chez l'expert comme chez l'apprenti. 
Cette typologie permet également à Jaffré de discuter la validité de l'hypothèse syllabique de Ferreiro, notamment pour l'acquisition de systèmes d'écriture différents de l'espagnol. Il s'interroge ainsi sur la réalité de certains codages syllabiques : «Dans le mot "glace", prononcé [glasø] et écrit $A E[\ldots]$, les lettres $A$ et $E$ sont-elles réellement des lettres-syllabes?» (ibid. : 46). Ses réserves-comme les nôtres - montrent que les propriétés linguistiques des langues et des systèmes d'écriture influencent les modalités du codage phonographique, jusqu'à déterminer les procédures déployées par les apprentis scripteurs. Dans la même perspective, Jaffré montre que les procédures qu'ils qualifient de « digrammiques » (par exemple : «boucherie » noté BOUCHRI, « tombe » noté TONB, " dans » noté DEN, etc.) "pourraient être le lieu d'un saut qualitatif faisant passer les enfants d'une attitude phonocentrée à une attitude graphocentrée » (ibid. : 46). Nous concluons, dans des termes équivalents, que la perception des variations phonographiques (par exemple [e] envisagé par trois graphèmes différents : $E R, E, E Z$, ou [wa] par un trigramme : $O U A$, ou un digramme : $O I$ ) manifeste une aptitude à analyser les informations graphiques, et donc une première prise de conscience de la complexité orthographique de notre écriture.

Enfin, des recherches plus récentes, menées par deux chercheuses québécoises, Montésinos-Gelet et Morin (2003), décrivent également les orthographes approchées de jeunes élèves plus âgés d'une année que les nôtres, à 6-7 ans. Dans leurs recherches, elles décrivent parfaitement la pluralité de leurs conceptions linguistiques dans une perspective variationniste, mais aussi leur capacité à analyser les éventuelles contradictions dans leur façon d'appréhender l'écrit, et à identifier les conséquences possibles de cette pluralité sur les productions en cours ou à venir, puis à vérifier, dans leur développement, les caractéristiques entourant les variations. Leurs conclusions croisent les nôtres dans le sens où les sujets de leur étude ont « des modes d'appréhension diversifiés de l'écrit (stratégie lexicale, médiation phonologique, stratégie syllabique) » (ibid.) et qu'ils ne se contentent pas de reproduire les formes selon un schéma développemental en étapes. Dans la même étude, elles concluent que la capacité des enfants à diversifier leurs stratégies graphiques implique, certes, la production d'erreurs, mais elles constituent surtout la trace visible d'une activité métalinguistique et la condition même du développement de futures compétences orthographiques. À partir de ces travaux, il ressort que « la conscience des stratégies [relatives à l'écrit] peut être bénéfique pour l'apprentissage de la lecture et de l'écriture » (ibid.) ; il faudrait alors pouvoir mesurer les effets, à terme, de ces pratiques d'écriture approchée sur l'acquisition de l'écriture en général et sur la maitrise des habiletés orthographiques en particulier (David et Morin, 2009 à paraitre).

$\mathrm{Au}$ terme de cette analyse, nous pouvons affirmer que la logique phonographique est décisive pour l'émergence de nouvelles compétences d'écriture, sans doute parce qu'elle implique une refonte des conceptualisations strictement sémiographiques de l'écrit, et une redistribution des connaissances et démarches de copie, selon des principes et avec des catégories plus explicitement linguistiques. Nous dirons alors qu'elle ouvre la réflexion des élèves et permet le repérage des variantes et irrégularités de notre système orthographique. Nous pouvons ainsi constater que les jeunes apprentis scripteurs changent d' « univers cognitif » (Bousquet et al., 1999); ils découvrent un autre mode d'expression écrite, une expression qui les conduit vers la reconnaissance de la variation phonographique, pour s'étendre au delà à d'autres dimensions de l'orthographe du français, notamment dans le domaine de la morphologie. 


\subsection{La morphographie : intégrer les caractéristiques de l'orthographe}

Au delà du principe alphabétique, les apprentis scripteurs doivent également traiter d'autres réalités linguistiques, et souvent compléter ou ré-analyser leurs connaissances antérieures pour envisager cette partie du système orthographique dans ses aspects grammaticaux ou lexicaux, dans une approche morphographique - et parfois morphosyntaxique - de faits de langue plus complexes à saisir. En effet, la mise en place des procédures sémiographiques et phonographiques ne suffit pas à rendre compte de la complexité de l'orthographe du français, profondément marquée par des catégorisations morphologiques. Les productions écrites des jeunes élèves de notre corpus témoignent de cette emprise et de la nécessité d'appréhender les phénomènes linguistiques correspondants.

D'autres travaux évoquent depuis peu l'émergence précoce d'une «conscience morphographique » ${ }^{(12)}$, peu ou prou équivalente à la « conscience phonologique » qui occupe les recherches sur l'acquisition de la lecture-écriture depuis plusieurs décennies. Sur le versant de la production écrite - qui nous concerne essentiellement ici-, nous pouvons observer le déploiement de recherches à la fois plus nombreuses et plus étendues ; même si leur base linguistique n'est pas toujours homogène ni clairement définie.

Cet intérêt récent pour la morphographie est lié à l'observation de comportement d'apprentis lecteurs ou scripteurs particulièrement sensibles aux configurations de lettres dans les mots, dans une perspective lexicale ou « dérivationnelle» (Pacton, 2003), mais aussi aux variations de certaines classes de mots (les noms, les adjectifs, et surtout les verbes) qui supportent des flexions ou des marquages catégoriels importants (le nombre, le genre, la personne, les temps-modes, etc.). Il reste que cette composante morphographique pose des problèmes spécifiques aux élèves francophones, et ce dès leurs premières tentatives d'écriture. De fait, dans leurs écrits, ils sont immédiatement confrontés à une orthographe qui s'est construite autour de procédés comme l'hétérographie en accumulant des marques distinctives : doublement des consonnes, ajout de finales muettes, multiplication de signes diacritiques et idéographiques, etc. Ils doivent aussi appréhender une information grammaticale décuplée par des marques multiples (par exemple, le pluriel des noms appliqué également aux adjectifs). Ils doivent enfin prendre en compte la complexité de systèmes et sous-systèmes de la langue - notamment la morphologie verbale (Blanche-Benveniste, 2002) - par des règles d'écriture difficilement inaccessibles. Cette évolution singulière de la morphographie du français a pour effet d'accroitre sensiblement le cout de la production orthographique, et surtout de son apprentissage.

Chez nos jeunes scripteurs, l'opacité de cette morphographie est immédiatement prise en compte dans des approches, certes, élémentaires et tâtonnantes, mais extrêmement révélatrices de la complexité de ce sous-système. L'expression du pluriel, par exemple, est à l'image de cette complexité. Elle se manifeste en apparence par un marquage régulier : 1'ajout d'un $-S$; elle engendre en fait une multitude de problèmes orthographiques, des problèmes qui s'élaborent au moins à deux niveaux :

(12) Les publications en langue française sont essentiellement l'œuvre d'équipes de psycholinguistes expérimentalistes : Gombert et al. (1997); Fayol et al. (2001); Colé et al. (2003); Largy et al. (2004); Pacton et al. (1999)...; et dans le paradigme de la linguistique génétique, avec des préoccupations didactiques conséquentes : Brissaud et al. (1999, 2001); Cogis (2001, 2004); Jaffré (2003a, 2003c); Sandon (2004) et nous-même, David (2003c). 
a) Au plan des opérations linguistiques ${ }^{(13)}$, les élèves doivent construire des catégories complexes - et donc très couteuses du point de vue cognitif -, puisqu'ils doivent tenir compte de critères spécifiques : i) le caractère inaudible des finales en $-S$, mais aussi en $-X$ des noms et des adjectifs, ou en $-N T$ de la majorité des verbes; ii) la redondance obligée de ces marques dans le cadre des accords syntaxiques au sein du groupe nominal, et du sujet sur le verbe ; iii) l'hétérogénéité de ce marquage morphosyntaxique, puisque qu'on peut avoir plusieurs morphèmes du pluriel pour une même classe de mots (un $-S$, un $-X$, et parfois $-\varnothing$ pour les noms).

b) Au plan strictement orthographique, avec une polyvalence morphémique démesurée, notamment avec ce $-S$ que les élèves doivent distinguer en fonction : i) de ces valeurs phonologiques, essentiellement celles du [s] et du [z], comme dans saison; ii) de la deuxième personne du singulier des verbes en $-E R$, la première et la deuxième des autres verbes; iii) de nombreux cas d'homophonie comme cours vs. cour, court...; iv) des temps-modes, notamment à la première personne du futur $j$ 'aimerai et du conditionnel $j$ 'aimerais; $v$ ) des dérivations et/ou des étymologies lexicales comme dans un temps gris, etc.

Bien évidemment, les jeunes élèves de notre étude sont loin d'appréhender des problèmes aussi complexes. Ils n'en approchent que les aspects les plus saillants et les plus immédiats... dans une logique morphographique spécifique. Les procédures observées témoignent ainsi d'opérations impliquant des représentations primitives du nombre et la mobilisation d'opérations de quantification linguistique plus ou moins adaptées. Nous montrons ainsi comment ces opérations correspondent à des procédés de catégorisation disponibles : la lexicalisation ou la grammaticalisation, à partir de critères essentiellement référentiels et sémantiques.

Cette composante morphographique apparait dans les EM de nos jeunes scripteurs et agit sur leurs productions écrites. La verbalisation des procédures correspondantes, attestées dans plusieurs EM, est décisive pour approcher cette composante de l'orthographe du français. Nous voyons en effet émerger des solutions morphographiques intelligentes, appliquées à des problèmes parmi les plus complexes à maitriser, et notamment :

a) l'inscription d'éléments lexicaux ou idéographiques graphiquement saillants, mais inaudibles dans l'énoncé oral, comme le $e$ muet de nombreux mots (Damien ( 5,1 ans) écrit «TOE » et quand on lui demande : «pourquoi tu as mis un $E$ ici », il répond «parce/ parce qu'il y a des $E$ à la fin des mots - pas toujours - mais des fois oui »);

b) l'insertion de morphèmes libres dont la place et l'importance sont désormais reconnues par repérage dans les énoncés oraux correspondants (Cyril ( 6,1 ans) ajoute «le 》 devant «papa» et explique «j' avais oublié le je l'ai mis en dessous... c'est le papa qu'on voit sur la photo c'est pas mon papa »);

c) la grammatisation, parfois originale, du nombre, qu'il s'agisse de l'expression du singulier (Medhi (5,7 ans) ajoute « $1 »$ devant «ani» (=ami) en expliquant «j'ai oublié de mettre $u n »)$, du pluriel ou du duel (Jean-Baptiste $(5,4$ ans) écrit «un AIAt / un AIAt» (= un chat / un chat) qu'il commente : « et j'ai écrit deux $A$ (en pointant les deux segments $A I A t$ ) parce que j'ai deux chats »); d) l'ajout de lettres muettes à valeur grammaticale, principalement le $S$ signi-

(13) Et essentiellement grammatical, du fait que le français, comme la plupart des langues indoeuropéennes, a privilégié un marquage morphologique au détriment d'une expression plus lexicale, présent dans de nombreuses langues africaines, amérindiennes ou asiatiques (Jaffré et David, 1999 ; Coulmas, 1989). 
fiant graphique de la pluralité (Nicolas (5,10 ans) écrit tout d'abord «les maison avec enfants » puis ajoute un $S$ à maison en expliquant « il manque un $C$ (en fait un $S$ ) - c'est parce qu'il y a les maisons (en insistant sur les) ») ;

e) la polyvalence de certains morphèmes dans le secteur de la morphologie verbale pour des homophones complets du type on vs ont (Cindy (6,4 ans) écrit avec deux biffures «les chouettes ent ensont » et justifie «Là j' ai dit [õ] avec $O-N$ - $T$ mais le $T$ on l'entend pas alors j'ai barré j'ai mis $O-N-S »$, puis se ravise « c'est avec un $T$-c'est comme ça que ça s'écrit (en montrant une affiche de la classe) ») ou 1'homophonie partielles des flexions en /E/ des verbes (Benjamin (5,6 ans) qui rature "ratovtr une histore» et réécrit «raqonté », en argumentant « parce que raqonté il faut barrer le $R$ - là j'ai écrit le $E$ de $f e ́-$ vrier... parce qu'il faut changer des fois $-E$ ou $E-R$ on peut mettre les deux »).

Concernant la notion de pluralité, nos observations confirment, s'il en était besoin, qu'elle n'est pas évidente à saisir et à traduire dans l'orthographe du français. Pour parvenir à noter le pluriel, les jeunes scripteurs procèdent par des catégorisations successives, des catégorisations qui se situent à plusieurs niveaux d'élaboration : des représentations extraites du monde environnant jusqu'aux opérations psycholinguistiques nécessaires à leur expression linguistique. Les séquences présentées ici confirment que la notion de pluralité et les opérations de détermination / quantification associées ne sont pas évidentes à mettre en relation dans l'écriture du français. Ces catégorisations passent bien souvent par une abstraction des données numériques immédiates : il faut tout d'abord opposer l'unique au pluriel et ensuite accepter de condenser dans cette représentation du pluriel toutes les expressions possibles de la pluralité ( « plein », «plusieurs », « beaucoup », ou bien « deux » comme « des milliers », mais aussi des collections ou des collectifs : « la vaisselle», « la classe », dont on peut dénombrer les éléments). Il faut en plus recourir à des solutions morphographiques aussi abstraites qu'arbitraires (Keller, 1998) et, dans le cas du français, difficilement saisissables, puisque peu lexicalisées et le plus souvent inaudibles. C'est la raison pour laquelle Jean-Baptiste (ci-dessus) teste des solutions possibles, si ce n'est probables, en jouant sur la duplication d'un même segment graphique pour signifier le duel, et peut-être le pluriel. De même, l'ajout d'un - $S$ final aux noms apparait pour Franck (ci-dessus) - dans des mouvements de réécriture certes différents - comme la réponse la plus évidente à un problème tour à tour référentiel, sémantique et morphologique. L'ajout de ce $-S$ grammatical - repéré directement ou suggéré par l'entourage - semble alors la solution la plus adéquate, dans un processus iconique (Dressler, 1995) de catégorisation, sans doute économique et probablement satisfaisant au plan linguistique.

Pour ces jeunes scripteurs - et plus encore dans leur cursus ultérieur d'apprentissage orthographique - nous constatons que ce - $S$ agit comme un morphème prototypique, un morphème qui sera parfois étendu à d'autres lexèmes, notamment ceux qui signifient une certaine pluralité (la*familles, la*classes, et même l'*échelles... « parce qu'elle à plusieurs barreaux »!). Cependant, avec ce marquage prototypique, rien n'est stabilisé, de nombreuses erreurs persistent au delà du cycle primaire, et même secondaire. La plupart des élèves - ceux de la présente étude, âgés de 5 à 6 ans, comme ceux qui orthographieront par la suite ce pluriel omettent de marquer tous les noms ${ }^{(14)}$.

Pourquoi donc, alors que la solution graphique du $-S$ semble être perçue et caté-

(14) Nous avons ainsi relevé qu'entre 5 et 8 ans, moins de $10 \%$ des noms exprimés au pluriel sont effectivement marqués en conséquence (Jaffré et David, ibid.). 
gorisée précocement - ce que confirment également des recherches expérimentales (Thévenin et al., 1999 ; Totereau, 1999 ; Cousin et al., 2003) - ne pouvonsnous observer une maitrise durable et homogène du pluriel orthographique, au moins pour le marquage des noms? L'explication ne peut être que cognitive ET linguistique. De fait, les apprentis scripteurs doivent d'une part intégrer ces calculs orthographiques dans la gestion des autres niveaux de construction du texte (Fayol, 1997), et d'autre part faire évoluer leur théorie du pluriel ; c'est-à-dire passer d'un simple marquage sémantique et/ou référentiel du pluriel à des calculs morphosyntaxiques plus complexes, mais aussi cognitivement plus couteux et certainement trop sophistiqués ${ }^{(15)}$.

Plus largement, et quelles que soient les solutions morphographiques trouvées ou les raisonnements invoqués, nous constatons que les EM avancées par les jeunes élèves de notre étude ne peuvent pas être - uniquement - organisées et légitimées par des savoirs enseignés; elles sont en fait déduites de leurs multiples expériences d'écriture. Nous montrons que nos jeunes scripteurs évoluent en verbalisant les cheminements cognitifs sous-jacents à la résolution des problèmes linguistiques rencontrés. De fait, leurs EM témoignent d'une démarche à la fois empirique, dynamique et heuristique. Les procédures morphographiques attestées ici ne surgissent pas du néant ; elles ne résultent pas non plus d'une imposition de listes ou de règles coupées de leur activité métalinguistique. Ces jeunes scripteurs construisent leurs connaissances par des organisations et des réorganisations successives qui suivent des trajectoires cohérentes et ajustées aux phénomènes linguistiques rencontrés. Ainsi, des solutions opérantes pour un problème factuel (par exemple la polyvalence phonogrammique, comme pour Benjamin) peuvent être réexaminées à la lumière d'autres problèmes (en l'occurrence l'homophonie des finales en /E/ des verbes) ${ }^{(16)}$. Cela passe, bien évidemment, par la capacité à raisonner, à accepter des variations, à étendre des règles ou au contraire à les restreindre ; toutes dispositions mises à jour dans les EM conduites dans les entretiens.

Ces fonctionnements cognitifs ajustés aux réalités de la langue et de son écriture montrent que les apprentis scripteurs ne sont jamais passifs dans leurs acquisitions. Ils parviennent à formuler des théories successives et évolutives de l'écriture en fonction des logiques qu'ils peuvent élaborer : un principe sémiographique fondamental, un système alphabétique économique et des caractéristiques morphographiques spécifiques. Pour cela, ils procèdent en construisant des savoirs nécessairement évolutifs et interactifs qui assurent le passage d'une logique à 1'autre (Gopnick et Meltzoff, 1997 ; Bousquet et al., 1999). Ce peut être 1'analyse d'un mot pour son insertion dans une phrase ou un texte. C'est également le traitement d'un même fait de langue dans des logiques déictique ou sémantique, phonographique, en fonction de réalités strictement graphique mais aussi de contraintes morpholexicales (Damien) ou morphogrammaticales (Medhi ou Cindy) et parfois morphosyntaxiques (Nicolas ou Benjamin).

(15) Nous pouvons aujourd'hui montrer que les accords en nombre des verbes, et plus encore des participes passés sont loin d'être définitivement acquis, à la fin de la scolarité obligatoire, à 16 ans (Brissaud et Chevrot, 2001), et au delà, puisque la réalisation de ces accords restent fragiles chez les adultes, même les plus experts (Jaffré, 2003b).

(16) C'est ce que montent des travaux aujourd'hui en expansion (Brissaud et Sandon, 1999 ; Brissaud et Chevrot, ibid) ; et les deux revues dirigées l'une par Jaffré et Brissaud (2003), l'autre par Brissaud et Totereau (2004), qui prouvent les difficultés persistantes des élèves jusqu'au cycle 3 de l'école primaire (10-11 ans) et au-delà au collège (jusqu'à 16 ans) à maitriser efficacement ce secteur de la morphologie verbale. 
À travers l'étude de ces premières manifestations de la morphographie, nous montrons également que les principes fondamentaux de l'écriture du français se présentent aux élèves dans toutes leurs composantes et qu'ils peuvent (doivent?) les appréhender de façon complémentaire. Nous constatons ainsi que les élèves ne se contentent pas d'enregistrer des formes écrites plus ou moins stabilisées ; ils les interrogent, les analysent, voire les recréent selon un processus souvent analogue à celui observé dans l'évolution des écritures humaines. Dans cette perspective, nous nous situons dans une perspective qui envisage l'activité cérébrale d'une façon dynamique qui s'oppose de fait à toute conception statique des apprentissages. Par la mise en œuvre de ces opérations psycholinguistiques, les élèves ne se contentent pas d'enregistrer les formes qu'on leur présente; ils les soumettent à leurs questionnements. Ils amorcent ainsi la mise en système des unités et catégories linguistiques rencontrées.

Au-delà, et pour que l'écriture soit réellement maitrisée, nous sommes désormais convaincus que les élèves doivent construire une conception multidimensionnelle de l'écrit, une conception qui leur permette de distinguer les phénomènes centraux et périphériques, de repérer les réponses productives ou " rentables » en terme de système, d'éliminer les solutions qui mènent à des impasses ou des « culs de sacs », pour reprendre l'expression que Dressler (1997) applique à l'acquisition de la morphologie des langues orales. Ces constats supposent alors que l'enseignement-apprentissage de l'écriture en général et de l'orthographe du français en particulier passe par une prise en compte réelle de la «faute » - au sens où Frei approche cette notion (1929/1993) - pour effectuer les différents marquages, ajuster leurs procédures et faire évoluer ces logiques sémiographique, phonographique et morphographique.

\section{Pratiquer l'autographie et les explications associées}

Le terme autographie sert à identifier un ensemble de pratiques permettant aux élèves d'écrire directement et de manière autonome des écrits, sans autre médiation que le recours à leurs propres habiletés scripturales et connaissances linguistico-graphiques, plus ou moins affirmées ou tâtonnantes. Ces pratiques s'opposent à certaines, essentiellement limitées à la copie ou au transport-copie (Rieben et Saada-Robert, 1993, 1997), et se superposent ou s'associent à d'autres comme la dictée à l'adulte (Clesse et Hébrard, 1977 ; David, 1991 ; Thévenaz-Christen et al., 2006).

Les pratiques autographiques que nous développons s'inspirent directement de travaux que nous avons exposés ici, d'une part dans la revue de travaux de notre première partie, d'autre part dans l'étude des écrits et EM que nous avons analysés dans la seconde partie. Cependant, cet ensemble d'études vise avant tout la formulation d'un modèle génétique de la production écrite, un modèle qui décrit les fonctionnements cognitifs des apprentis scripteurs et les procédures psycholinguistiques propres à l'appropriation des principes d'écriture de leur langue. De fait, dans ces travaux les implications didactiques ne sont pas toujours présentes et explicitement développées ${ }^{(17)}$. Pourtant, nous pouvons aujourd'hui montrer que cette pratique démultiplie les capacités langagières des élèves, en général, et une prise de conscience des composantes linguistiques orales et écrites. Les élèves de

(17) Voir cependant l'ouvrage publié récemment par Montésinos-Gelet et Morin (2006) ou le nôtre, David (2009, à paraitre). 
ces travaux - déjà apprentis lecteurs et scripteurs - approchent ainsi les logiques sous-jacentes au système écrit, et parviennent à les mettre en œuvre dans des écrits originaux.

À travers les différentes expériences d'écriture et les procédures émergentes, et restituées pour partie ici, nous prouvons leur capacité à saisir l'économie du système alphabétique du français, mais aussi de ses caractéristiques morphologiques, qu'ils testent et approchent à travers des écritures plus ou moins normées. Plus encore, lorsqu'ils commentent et expliquent les procédures ainsi testées, ils montrent leur disponibilité à comprendre et à employer ces procédures sémio-, phono- et morphographiques, mais aussi à les utiliser de façon plus ajustée et plus systématique dans de futurs écrits. Nos travaux révèlent ainsi des phénomènes d'apprentissage, voire d'auto-apprentissage (Fayol, 2006), singuliers qui offrent aux jeunes scripteurs de véritables situations de « résolution de problèmes » d'écriture, analogues à ceux découverts dans d'autres enseignements-apprentissages, notamment dans le domaine mathématique.

Au delà, ces recherches psycholinguistiques appliquées à la production écrite et aux auto-explications conséquentes montrent que les jeunes élèves sont disponibles pour des apprentissages orthographiques révélant la complexité du français écrit. Nous avons ainsi montré qu'ils recourent à des procédures ajustées aux logiques, principes, propriétés de cette écriture, et parviennent à trouver des solutions linguistico-graphiques soit par l'application de raisonnements comme celui de la dérivation lexicale (gros, avec un $s$ parce que grosse, grosseur...), soit par le repérage de régularités souvent liées à des fréquences d'emploi (la position et la fréquence du eau en finale des mots en $/ \mathrm{O} /$ )... alors que des élèves plus âgés du cycle secondaire, dont nous avons décrit ailleurs les écrits scolaires (David, 2005b), ne parviennent toujours pas à les employer de manière efficiente. Nous pensons dès lors ouvrir de nouvelles perspectives didactiques pour des élèves dont les performances orthographiques semblent se déliter (Manesse et Cogis, 2007), ou pour le moins se distendre dans des pratiques plus diversifiées et moins normées : les écritures informatiques notamment, décrites par Anis $(1998,2001)$ ou nous-même (David et Gonçalvez, 2007). Nous pourrions également nous inspirer de ces pratiques combinant l'autographie et les commentaires métagraphiques pour aider des rédacteurs adolescents et adultes, plus ou moins identifiés comme illettrés (Lucci et Millet, dir., 1994).

De fait, les auto-explications orthographiques formulées par les jeunes élèves décrits dans nos travaux ont une valeur heuristique déterminante pour les apprentissages de l'écrit. Reste à les organiser dans des dispositifs d'apprentissage spécifiques, dans des activités « intelligentes » qui permettent aux élèves de repérer ces fonctionnements orthographiques et à les construire dans des ensembles plus ou moins systémiques mais nécessairement inscrits dans la dynamique de l'écriture. Ces apprentissages en situation n'excluent pas, bien évidemment une étude réfléchie des formes conventionnelles de l'écrit. De fait, la recherche de Rieben et al, (2005) prouve que les écritures approchées, à condition qu'elles soient validées ou corrigées par l'enseignant, débouchent sur des acquisitions procédurales supérieures à toutes les autres pratiques évoquées ici, et notamment le simple transport-copie. Nous ajouterons, à l'issue des travaux rapportés ici - et d'autres en cours ou à venir (David et Morin, 2009) - que la découverte des principes de l'écriture du français n'assure pas, à elle seule, la pérennité des savoirs orthographiques. Car, pour que ces savoirs émergents débouchent sur des connaissances stabilisées et transférables, il convient de les organiser dans des apprentissages 
complémentaires visant plus systématiquement la mise en place de routines ou d'automatismes orthographiques, et leur réinvestissement dans des situations de production textuelle (Allal et al., 2001).

\section{Références bibliographiques}

Allal, L., BétriX-Koehler, D., Rieben, L., Rouiller-Barbet, Y., SAAdAROBERT, M.\& WEGMULLER, E. (2001) : Apprendre l'orthographe en produisant des textes, Fribourg : Éditions universitaires de Fribourg.

AngOujard, J.-P. (1997) : Théorie de la syllabe. Rythme et qualité, Paris : CNRS éditions.

AnIS, J. (1998) : Texte et ordinateur. L'écriture réinventée? Bruxelles : De Boeck Université.

- (dir.) (2001) : Parlez-vous texto ? Paris : Le Cherche midi.

BESSE, J.-M. (1990) : «L'enfant et la construction de la langue écrite », Revue française de pédagogie, 90, 17-22.

Besse, J.-M., Rouzaire, M. \& VeilleuX-Sourd, N. (1999) : « L'entrée dans la phonétisation de l'écriture : le rôle des interactions sociales », Les Dossiers des sciences de l'éducation, 1, 69-80.

BISSEX, G.L. (1980) : GNYS AT WRK : A Child Learn to Read and Write, Cambridge (Mass.) : Harvard University Press.

BlANCHE-BENVENISTE, C. (2002) : « Structure et exploitation des verbes en français contemporain », Le français aujourd'hui, 139, 13-22.

Bousquet, S., Cogis, D., DucArd, D., MAssonnet, J. \& JAFFrÉ, J.-P. (1999) : " Acquisition de l'orthographe et mondes cognitifs », Revue française de pédagogie, 126, 23-37.

BRISSAUD, C. \& CHEVROT, J.-P. (2001) : « Acquisition de la morphographie entre 10 et 15 ans : le cas du pluriel des formes verbales en /E/ », Verbum, XXII-4, 425-439.

BRISSAUD, C. \& SANDON, J.-M. (1999) : «L'Acquisition des formes verbales en /E/ à l'école élémentaire et au collège, entre phonographie et morphographie ", Langue française, 124, 40-57.

ByBeE, J.L. (2001) : Phonology and Language Use, Cambridge (UK) : Cambridge University Press.

CATACH, N. (1989/1995, $3^{\mathrm{e}}$ édition) : L'Orthographe française, Paris : Nathan.

CHOMSKY, C. (1971) : «Write first, read later», Childhood Education, 47, 296-299. - (1975) : «Invented Spelling in the open Classroom », Child Language Today, Word, $\mathrm{n}^{\circ}$ spécial, 499-518.

CLARKE, L.K., (1988) : « Invented versus traditional spelling in first grader's writings : Effects on learning to spell and read», Research in the Teaching of English, 22, 281-309.

CLESSE, C. \& HÉBRARD, J. (1977) : « Rôle du parler dans l'apprentissage de l'écrit », in L. Lentin (éd.), Du parler au lire. Paris : ESF.

Cogis, D. (2001) : «Difficultés en orthographe : un indispensable réexamen », Revue française de linguistique appliquée, VI-1, 47-61. 
- (2004) : «Une approche active de la morphographie. L'exemple d'une séquence sur l'accord de l'adjectif », LIDIL, 30, 73-86.

Colé, P., Marec-Breton, N., Royer, C. \& Gombert, J.-E. (2003) : « Morphologie des mots et apprentissage de la lecture », Rééducation orthophonique, 213, 57-76.

Coulmas, F. (1989): The Writing Systems of the World, Oxford: Basil Blackwell.

Cousin, M.-P., LARGY, P. \& F AYOL, M. (2003) : «Produire la morphologie flexionnelle du nombre nominal : étude chez l'enfant d'école primaire ", Rééducation orthophonique, 213, 115-129.

D AVID, J. (1991) : «La dictée à l'adulte ou comment de jeunes enfants oralisent l'écrit », Études de Linguistique Appliquée, 81, 7-20.

- (2003a) : «La dimension orthographique dans les écrits des jeunes enfants », Les Dossiers des Sciences de l'éducation de l'université Toulouse-Le Mirail, 9, 29-39.

- (2003b) : «Linguistique génétique et acquisition de l'écriture », Faits de langue, 22, 37-45.

- $(2003 \mathrm{c})$ : « Les procédures orthographiques dans les productions écrites des jeunes enfants », Revue des sciences de l'éducation (Québec), vol XXIX$1,137-158$.

— (2005a) : «Catégories langagières et cognitives. L'exemple des premiers apprentissages de l'écriture ", Le français aujourd'hui, 151, 81-94.

— (2005b) : «L'écriture des collégiens de banlieue, entre pratiques singulières et normes scolaires », in M.-M. Bertucci \& V. Houdart-Mérot (dir.), Situations de banlieues : enseignement, langues, cultures, Paris : Institut national de recherche pédagogique, coll. "Éducation, politiques, sociétés».

— (2006) : "Quelles pratiques de production écrite à l'école maternelle ?», La Lettre de l'AIRDF, 39, 23-28.

- (2009, à paraitre): Les Écritures approchées : quelles pratiques au cycle 2 de l'école primaire? Paris : Hatier.

D AVID, J. \& GONÇALVES, H. (2007) : «L'écriture électronique, une menace pour la maitrise de la langue ?», Le français aujourd'hui, 156, 39-47.

D AVID, J. \& JAFFRÉ, J.-P. (1997) : « Le rôle de l'autre dans les procédures métagraphiques », Recherches, 26, 155-168.

DAVID, J. \& MORIN, M.-F. (2008) : «Écritures approchées : des procédures métagraphiques des jeunes apprentis-scripteurs aux pratiques d'apprentissage », in J. Dolz \& S. Plane (dir.), Formation des enseignants et enseignement de la lecture-écriture. Recherches sur les pratiques, Namur: Presses universitaires de Namur, coll. « Diptyque » 13.

— (2009, à paraitre) : «L'apprentissage de la lecture-écriture en maternelle (5-6 ans) à travers les explications métagraphiques de jeunes scripteurs français et québécois ", Actes du $3^{\mathrm{e}}$ Colloque international de 1'ABLF, Interactions entre le lire et l'écrire. Bruxelles.

DAVID, J., BRISSAUD, C. \& GUYON, O. (2006) : «Apprendre à orthographier les verbes : le cas de 1'homophonie des finales en /E/ », Langue française, 151, 109-126.

DRESSLER, W.U. (1995) : «Interactions between iconicity and other semiotic parameters in language », in R. Simone (ed.), Iconicity in language, Amsterdam : John Benjamins.

— (ed.) (1997) : Studies in Pre-and Protomorphology, Wien (Aut.) : Verlag der Österreichischen Akademie der Wissenschaften. 
FAYOL, M. (1997) : Des Idées au texte, psychologie cognitive de la production verbale, orale et écrite, Paris : Presses universitaires de France.

— (2006) : "L'orthographe et son apprentissage », in Actes des journées de l'observatoire, «Enseigner la langue : orthographe et grammaire », Paris : Observatoire national de la lecture.

FAYOL, M. \& TOTEREAU, C. (2001) : «Learning the written morphology of plural in written french », in L. Tolchinsky (ed.), Studies in Writing, 8. Developmental Aspect in Learning to Write, Dortrecht (Nederlands) : Kluwer Academic Publishers.

FERREIRO, E. (1988) : «L'écriture avant la lettre », in H. Sinclair (éd.), La Production de notations chez le jeune enfant, Paris : Presses universitaires de France (version revue et corrigée dans E. Ferreiro (2000). L'Écriture avant la lettre. Paris : Hachette).

FERREIRO, E. \& GOMEZ-PALACIO, M. (1988) : Lire-écrire à l'école : comment s'y apprennent-ils? Analyse des perturbations dans les processus d'apprentissage de la lecture et de l'écriture, Lyon : CRDP.

FERreiro, E. \& TEBERosky, A. (1979) : Los Sistemas de Escritura en el Desarrollo del Nino, Mexico : Siglo XXI (trad. anglaise : Literacy Before Schooling, Exeter (N.H.) and London: Heinemann Educational Books, 1982).

FiJALKOW, J. \& FiJALKOW, É. (1991b) : «L'écriture inventée au cycle des apprentissages. Étude génétique », Les Dossiers de l'Éducation, 18, 125-167.

FiJAlKow, J. (1993) : «Entrer dans l'écrit : des niveaux successifs », in G. Chauveau, M. Rémond \& É. Rogovas-Chauveau (éds.), L'Enfant apprenti lecteur. Paris : I.N.R.P. - L'Harmattan.

FranÇOIS, F., Hudelot, C. \& SABEAU-JouAnnet, E. (1984) : Conduites linguistiques chez le jeune enfant, Paris : Presses universitaires de France.

FREI, H. (1929/1993) : La Grammaire des fautes, Genève-Paris : Slatkine Reprints.

Gombert, J.-É., BryAnT, P. \& WARRICK, N. (1997) : «Les analogies dans l'apprentissage de la lecture et de l'orthographe », in L. Rieben, M. Fayol \& C.A. Perfetti (éds.), Des Orthographes et leur acquisition, Lausanne : Delachaux \& Niestlé.

Gopnick, A. \& Meltzoff, A.N. (1997) : Words, Thoughts, and Theories, Cambridge (Mass.) : Cambridge University Press.

GoswAMI, U. (1988) : « Children's use of analogy in learning to spell », British Journal of Developmental Psychology, 6, 21-33.

JAFFRÉ, J.-P. (1992) : «Le traitement élémentaire de l'orthographe : les procédures graphiques », Langue française, 95, 27-48.

- (2003a) : «La morphographie du français : un cas sémiographique », Rééducation orthophonique, 213, 13-25.

- (2003b) : «L'écriture \& les nouvelles technologies. Ce que les unes nous apprennent de l'autre. S'écrire avec les outils d'aujourd'hui / Points de vue croisés », Communication à la journée d'études «Réseaux humains / Réseaux technologiques », 31 mai- $^{\text {er }}$ juin 2002, MSHS Poitiers.

- (2003c) : «La linguistique et la lecture-écriture : de la conscience phonologique à la variable “orthographe” ", Revue des sciences de l'éducation (Québec), vol XXIX-1, 37-49.

JAFFRÉ, J.-P. \& DUCARD, D. (1996) : «Approches génétiques et productions graphiques », Études de linguistique appliquée, 101, 87-98.

JAFFRÉ, J.-P. \& D AVID, J. (1999) : «Le nombre : essai d'analyse génétique », Langue française, 124, 7-22. 
JAFFRÉ, J.-P., BoUSQUET, S. \& MASSONNET, J. (1999) : « Retour sur les orthographes inventées ", Les Dossiers des sciences de l'éducation, 1, 39-52.

JAFFRÉ, J.-P. \& BRISSAUD, C. (dir.), (2006) : « Morphographie et homophones verbaux », Langue française, 151.

KARMiloff-S Mith, A. (1992) : Beyond Modularity. A Developmental Perspective on Cognitive Science, Cambridge (Mass.) : The MIT Press.

KELLER, R. (1998) : A Theory of linguistic signs, Oxford (UK) : Oxford University Press.

LARGY, P. \& COUSIN, M.-P. (2004) : «Apprendre implicitement le - $s$ du pluriel », in A. Piolat (dir.), Écriture. Approches en sciences cognitives, Aix-en-Provence : Presses universitaires de Provence, coll. «Langues et écritures ».

LuCCI, V. \& Millet, A. (dir.) (1994) : L'Orthographe de tous les jours. Enquête sur les pratiques orthographiques des Français, Paris : Champion.

MAnesse, D. \& Cogis, D. (2007) : Orthographe, à qui la faute?, Paris : E.S.F.

MONTÉSINOS-GELET, I. \& MORIN, M.-F. (2003) : «S'approcher de la norme orthographique en 1ère année du primaire : qu'en est-il de la pluralité des conceptions linguistiques? », Archives de Psychologie, 69, 159-176.

- (2006) : Les Orthographes approchées. Une démarche pour soutenir l'appropriation de l'écriture au préscolaire et au primaire, Montréal (Québec) : Chenelières « Éducation».

MORIN, M.-F. (2004a) : «Les niveaux d'explicitation des connaissances sur la morphographie du nombre au début du primaire », LIDIL, 30, 55-72.

— (2004b) : «Comprendre et prévenir les difficultés en écriture chez le jeune enfant : orthographes approchées et les commentaires métagraphiques », in J.-C. Kalubi \& G. Debeurme (dir.), Identités professionnelles et interventions scolaires. Contextes de formation de futurs enseignants, Sherbrooke (Québec-Can.) : Éditions du CRP.

— (2005) : «Declared knowledge of beginner writers », L1- Educational Studies in Language and Literature, 5, 385-401.

PACTON, S. (2003) : «Morphologie et acquisition de l'orthographe : état des recherches actuelles », Rééducation orthophonique, 213, 27-55.

PACton, S., FAyOL, M., LonjarRet, D. \& Dieudonné, D. (1999) : « Apprentissage implicite et orthographe. Le cas de la morphologie », Rééducation orthophonique, 200, 91-100.

PEYTARD, J. (1970) : « Oral et scriptural : deux ordres de situations et de descriptions linguistiques », Langue française, 6, 35-47.

RAVID, D. (2001) : « Learning to spell in Hebrew : Phonological and morphological factors », Reading and Writing, 14, 459-485.

— (2006) : «Hebrew orthography and literacy», in R. Malatesha Joshi \& P.G. Aaron (éds.), Handbook of orthography and literacy, Mahwah (N.J.) : L. Erlbaum Associates Publishers.

READ, C. (1971) : «Pre-school children's knowledge of english phonology », Harvard Educational Review, 41, 1-34.

- (1986) : Children's Creative Spelling, London : Routledge \& Kegan Paul.

RIEBEN, L. \& SAADA-ROBERT, M. (1997) : «Étude longitudinale des relations entre stratégies de recherche et stratégies de copie de mots chez des enfants de 56 ans », in L. Rieben, M. Fayol \& C.A. Perfetti (éds.), Des Orthographes et leur acquisition, Lausanne : Delachaux \& Niestlé.

Rieben, L., Ntamakiliro, L., Gonthier, B. \& FAYOL, M. (2005) : « Effects of 
Various Early Writing Practices on Reading and Spelling », Scientific Studies on Reading, 9(2), 145-166.

RitTle-Johnson, B. \& SiEgLER, R. (1999) : « Learning to spell : Variability, choice, and change in children's strategy use », Child Development, 70, 332-348.

RUBIN, H. \& EBERHARDT, N.C. (1996) : «Faciliting invented spelling through language analysis instruction : An integrated model », Reading and Writing, 8 (1), 27-43

SAADA-ROBERT, M. \& RIEBEN, L. (1993) : «Évolutions des stratégies d'écriturecopie et unités graphiques du français », Études de linguistique appliquée, 91, 84-96.

SANDON, J.-M. (2004) : «Le traitement de la finale du mot graphique par l'apprenti. Un seuil cognitif entre apprentissage et expertise », LIDIL, 30, 169-182.

SEYMOUR, P.H.K. (1993) : «Un modèle de développement orthographique à double fondation », in J.-P. Jaffré, L. Sprenger-Charoles \& M. Fayol, Lecture-écriture : acquisition. Les Actes de la Villette, Paris : Nathan.

— (1997) : «Les fondations du développement orthographique et morphographique », in L. Rieben, M. Fayol \& C.A. Perfetti (éds.), Des Orthographes et leur acquisition, Lausanne : Delachaux \& Niestlé.

Teberosky, A., Tolchinsky-Landsmann, L. et al. (1993) : « Segmentation phonologique et acquisition de l'écriture en castillan, catalan et hébreu », Études de linguistique appliquée, 91, 48-59.

Thévenaz-Christen, T., Claude, J. \& Dombre, C. (2006) : « Activités langagières et métalangagières au cours d'une séquence de dicte à l'adulte », Langage et pratiques, 38, 34-45.

Thévenin, M.-G, Totereau, C., Fayol, M. \& Jarousse, J.-P. (1999) : « L'apprentissage / enseignement de la morphologie écrite du nombre en français », Revue française de pédagogie, 126, 39-52.

TOTEREAU, C. (1999) : «Apprendre la morphologie du nombre à l'écrit en français » Rééducation orthophonique, 200, 101-113.

Totereau, C. \& Brissaud, C. (2006) : «Acquisition des flexions verbales homophones en /E/: une étude longitudinale du CE2 au CM2 », Rééducation Orthophonique, "La morphologie : acquisition et mise en æeuvre », 225, 59-73.

TREIMAN, R. (1989) : «Le rôle des unités intrasyllabiques dans l'apprentissage de la lecture ", in L. Rieben \& C. Perfetti (éds.), L'Apprenti lecteur. Recherches empiriques et implications pédagogiques, Neuchâtel-Paris : Delachaux \& Niestlé.

— (1993b) : Beginning to Spell : A Study of firt-grade Children, New York : Oxford University Press.

TREIMAN, R. \& Bourassa, D. (2000) : « The development of spelling skill», Topics in Language Disorders, 20-3, 1-18.

Treiman, R., Bowey, J.A. \& BourassA, D. (2002) : «Segmentation of spoken words into syllabes by English-speaking children as compared to adults », Journal of Experimental Child Psychology, 63, 213-238. 